Running Head: Intuitions and Reducing Discrimination

Article in press, Personality and Social Psychology Bulletin

Misplaced Intuitions in Interventions to Reduce Attractiveness-Based Discrimination
Jordan R. Axt*1,2
Juanyu Yang ${ }^{1}$
Harshadaa Deshpande ${ }^{1}$
${ }^{1}$ McGill University
${ }^{2}$ Project Implicit

Corresponding Author Contact:

Department of Psychology, McGill University

2001 McGill College Ave

Montreal, Quebec, Canada, H3A 1G1

Email: jordan.axt@mcgill.ca 
Authors' Note: All data and study materials are available at the project page on the Open Science Framework (https://osf.io/uxrcs/). Studies 2a-2b were previously presented in the second author's undergraduate honours thesis. This research was supported by FRQSC grant number 2021-NP-282404.

Declaration of Conflicting Interests: This research was partly supported by Project Implicit. J. R. Axt is Head of Data and Methodology for Project Implicit, Inc., a nonprofit organization with the mission to "develop and deliver methods for investigating and applying phenomena of implicit social cognition, including especially phenomena of implicit bias based on age, race, gender, or other factors." There are no other potential conflicts of interest with respect to authorship or the publication of this article. 


\begin{abstract}
Individuals and organizations are increasing efforts to address discrimination. Non-experts may lack awareness of or are resistant to scientifically-informed strategies for reducing discrimination, instead relying on intuition. Five studies investigated the accuracy of nonexperts' intuitions about reducing discrimination concerning physical attractiveness. In Studies 1a-1c $(N=902)$, participants predicted the effectiveness of six interventions to reduce attractiveness-based favoritism on a judgment task. Studies $2 \mathrm{a}-2 \mathrm{~b}(N=6,292)$ investigated the effectiveness of these interventions. While two interventions reduced discrimination, intuitions were poorly aligned with actual results; fewer than $1 \%$ of participants identified the combination of interventions that did versus did not impact judgment, and responses were more likely to be below than above chance when predicting each intervention's effectiveness. Though follow-up work should investigate the accuracy of intuition in other forms of discrimination, the current results further stress the need for greater development and adoption of evidence-based strategies for combatting discrimination.
\end{abstract}

Keywords: discrimination, bias, judgment, decision-making, intuition, physical attractiveness Word count: 150 


\section{Misplaced Intuitions in Interventions to Reduce Attractiveness-Based Discrimination}

Compared to stereotypes, which are cognitive associations between groups and attributes, and prejudices, which are affective attitudes towards groups, discrimination is the behavioral output that generates and sustains disparities in real-world outcomes (Fiske, 1998). Field and laboratory research shows that discrimination is pervasive in the general population and among professionals (e.g., Crosby et al., 1980; Dovidio \& Gaertner, 2000; Moss-Racusin et al., 2012).

One prominent form of discrimination is based in physical attractiveness. Physical attractiveness is unlike other forms of discrimination, such as those based in race or gender, as attractiveness does not have a strong ingroup versus outgroup component; in fact, prior work suggests that people have minimal insight into how others rate them in terms of attractiveness (Kenealy et al., 1991). At the same time, people with higher or lower levels of physical attractiveness share a characteristic that has repeatedly been shown to impact real-world treatment (Hosoda et al., 2003). For instance, physically attractive people are more likely to receive job offers and promotions (Maestripieri et al., 2017), are less likely to be fired (Commisso \& Finkelstein, 2012). More attractive people also attend more coveted academic programs and achieve greater scholarly impact (Hale et al., 2021). Higher levels of physical attractiveness have even been associated with more moderate criminal sentences (Downs \& Lyons, 1991). Favoritism towards more physically attractive people persists even among more experienced evaluators (Marlowe, Schneider \& Nelson, 1996). In all, the benefits of being physically attractive are present in many highly consequential areas of life.

Increasingly, individuals and organizations are tasked with addressing and reducing biases in judgment and evaluation that are related to physical attractiveness. For example, prominent businesses like LinkedIn (Recruitics, 2021) and Twitter (Shankland, 2021) have 
sought changes in their platforms to reduce attractiveness-based discrimination. Ideally, in these cases, organizations could look to academic research for what changes or interventions to implement, but this approach can be significantly complicated by studies relying on different populations, procedures and outcomes. More importantly, while past research has identified several bias-reducing interventions in a variety of domains that have been successful in lab contexts, the current research literature lacks evidence for a suite of flexible intervention strategies that can be applied to reducing multiple forms of discrimination in both the lab and the field (e.g., Chang et al., 2019; Dobbin et al., 2007), though exceptions to this claim include the practice of "blinding" applications by completely removing irrelevant social information (Goldin \& Rouse, 2000).

Without proper scientific guidance, decision-makers may have to rely on their intuitions about how to debias judgments that are possibly influenced by physical attractiveness. However, using intuition (i.e., instinctive feelings not fully informed by empirical evidence) may prioritize solutions that feel impactful, but could be unlikely to successfully address attractiveness-based discrimination given prior work on people's limited ability to accurately predict the causes of various psychological processes (e.g., Nisbett \& Wilson, 1977). As a result, many strategies to reduce biased behavior may fall short of expectations, disappointing those who oversaw their implementation and allowing discrimination to persist.

In fact, individuals may have poor awareness for how to reduce discrimination, as suggested by prior work highlighting a general lack of accurate insight or misplaced confidence in irrelevant inputs for decision-making. For example, 47\% of HR professionals in a recent sample agreed with the notion that handwriting analyses are useful in predicting job success (Fisher et al., 2020), though prior studies have found no consistent association between 
handwriting and performance (Schmidt \& Hunter, 1998; Simner \& Goffin, 2003). Moreover, HR professionals show a reliance on intuition in strategies for determining employee evaluation, despite the availability of clear strategies for improving judgment. For example, while structured interviews have been shown to reduce biases in hiring and promotion (McDaniel et al., 1994), a survey of hiring managers still found a strong preference for unstructured over structured interviews ( $d=1.37$; van der Zee et al., 2002).

While such work reveals areas in which individuals, even those with supposed expertise in interpersonal evaluation, suffer from inaccurate intuitions about how to best improve judgment, no studies have extended this issue to addressing discrimination specifically. And, while many studies suggest high levels of inaccuracy in judgments relying on intuition, the personal importance that many individuals attach to appearing nonprejudiced (e.g., Plant \& Devine, 1998) may lead to a heightened motivation for accuracy in this context, creating greater insight in the domain of discrimination relative to those covered in prior work.

To explore this issue, we asked participants to view six bias-reducing interventions based on prominent strategies in the academic literature, and to predict how those interventions would fare when improving performance on a task known to reliably create discrimination based on physical attractiveness. Next, we tested the actual effectiveness of these interventions through a large-scale comparative test using the same outcome measure. Given the consequences of such discrimination, data concerning whether non-experts have any insight into the effectiveness of different bias-reduction interventions carries practical and theoretical implications.

From a practical perspective, results finding a lack of insight into what interventions reduce attractiveness-based discrimination will only strengthen calls for greater development and testing of scientifically-backed interventions. Theoretically, such results can add to existing 
models of biased judgment. For example, the Wilson and Brekke (1994) model of "mental contamination" posits that bias can be avoided when decision-makers have the ability to properly adjust their responses. If participants show high levels of accuracy in predicting interventions that do or do not reduce discrimination, it would suggest introspective clarity in understanding what changes or strategies allow people to effectively adjust their behavior when confronted with the possibility of bias. Such results would then suggest that bias primarily arises from an inability to implement these changes (e.g., due to a lack of awareness, fatigue, or distraction).

Conversely, low accuracy in identifying effective interventions would illustrate that just as people often lack insight into information that biases their judgment, so too do they lack insight into the strategies that remove the influence of such information. In this case, biased behavior would arise not only among people who lack awareness into how their behavior is being impacted, but also among many of those who successfully notice their decisions may be biased but then employ ineffective strategies in reducing this bias.

\section{Measuring Discrimination in Social Judgment}

To test intervention effectiveness, participants completed a measure called the Judgment Bias Task (JBT; Axt et al., 2018). In a JBT, people evaluate different profiles with relevant or irrelevant criteria for a particular outcome. In one version of the JBT, participants are on the selection committee for a hypothetical academic honor society and are given a series of applicant profiles to accept or reject. Profiles contain relevant information, such as GPA and interview score, and irrelevant social information, such as applicant political affiliation, race, or here a photo signaling physical attractiveness.

Bias is often measured using a within-subjects approach by adopting a Signal Detection Theory analysis in comparing the criterion values for accepting applicants from the two social 
groups represented in the task (e.g. more versus less physically attractive people); a lower criterion value for an acceptance decision towards one group over another group indicates that despite the two groups having the same overall level of qualifications, applicants from one group were held to a more lenient standard for admission. Furthermore, when coupled with self-report measures, those participants who after the task reported not wanting to use social information in their judgments or reported having not used social information in their judgments, still showed biases in the task on average, indicating that the JBT can detect discrimination that exists outside of conscious awareness or control (Axt et al., 2018).

The present work focused specifically on biases related to physical attractiveness, as it was a form of bias that is produced consistently on the JBT (Axt, Casola \& Nosek, 2019; Axt \& Lai, 2019). Given the consistent evidence of appearance-based discrimination across many consequential contexts, physical attractiveness is a sensible domain of study for investigating the accuracy of intuitions about bias reduction. Here, we use a primarily between-subjects approach by testing the effectiveness of various intervention strategies for reducing the degree of discrimination exhibited on the JBT.

\section{Candidate Interventions}

We identified six interventions that were previously found to either reduce bias in judgment or improve reasoning, though not all have been applied to social judgment or physical attractiveness specifically.

Accountability. Accountability refers to the expectation that one may need to justify their beliefs, attitudes, or actions to others (Lerner \& Tetlock, 1999). There are different methods for imposing accountability, including though external evaluation, reason-giving, or the mere presence of another person. Multiple studies have found that imposing accountability can 
significantly reduce judgment biases (e.g., Palmer \& Feldman, 2005; Paolini et al., 2009;

Vieider, 2009), but only if accountability was heightened before completing the relevant task and if biases were attributed to the failure to use all relevant cues and attend to one's decision processes (e.g., Kennedy, 1993). In particular, accountability may create impression management concerns that trigger preemptive self-criticism that partly shields people from mindlessly applying simple heuristics (Lerner \& Tetlock, 1999).

Educating about confirmation bias. Confirmation bias is the tendency to seek out information consistent with existing beliefs. In the context of the JBT, confirmation bias may lead participants to focus on the relative strengths of more physically attractive candidates while ignoring weaknesses. Understanding and suppressing confirmation bias may then be helpful in reducing bias. A recent study (Sellier et al., 2019) found that an effortful intervention centered around educating participants about confirmation bias reduced reasoning errors on a complex business case, though other instances of alerting people to biasing information have not always been successful (e.g., Jaeger et al., 2020). Still, learning about confirmation bias before completing the JBT could help reduce discrimination on the task.

Delay. Rational, unbiased decision-making takes time, and allocating less time than is needed for judgment causes "time stress", which can increase reliance on heuristics and lessen the amount of information considered (Ariely \& Zakay, 2001; Chandler \& Pronin, 2012). In prior work, greater time pressure when completing the First-Person Shooter Task (FPST), a decision-making task where participants try to quickly identify guns or harmless objects in the hands of Black or White targets, caused participants to exhibit stronger race-based discrimination (Axt \& Lai, 2019). A follow-up study found that the reverse strategy-- requiring a 4500 ms delay 
in responding-- increased accuracy and reduced discrimination on the JBT (Axt \& Lai, 2019), with similar results also being found in the FPST (Correll et al., 2015).

Implementation Intentions. Implementation intentions are a self-regulatory strategy that uses "if-then" statements to achieve certain goals, and rehearsing such statements has been shown to improve decision-making. In one study (Mendoza et al., 2010), participants completed an FPST, but beforehand half rehearsed a distraction-inhibiting implementation intention ("If I see a person, then I will ignore his race!'). Participants who rehearsed these implementation intentions showed less racial bias on the task, an outcome that is possibly explained by facilitating the automatic initiation of goal-directed behaviors without conscious reflection (Amodio et al., 2007). Implementation intentions have also been shown to be effective, at least in the short term, for reducing other instances of more automatic social biases (e.g., Lai et al., 2014; Webb et al., 2012).

Objectivity. Committing to unbiased behavior and listing desired judgment criteria in advance may create a need for greater consistency between one's values and actions. Some evidence for the effectiveness of this approach can be found in Uhlmann and Cohen (2005). Here, participants evaluated a candidate for police chief that was either male or female, and the applicants' strengths were either described as being streetwise or well-educated. In control conditions, results found that the hiring criteria emphasized in judgment (either being streetwise or well-educated) were consistent with whatever trait was paired with the male applicant, meaning participants favored the male applicant regardless of actual qualifications. However, when participants were asked to rate the importance of each criteria prior to learning the applicants' gender, there was no significant difference in the evaluation of male and female applicants. A similar approach that guides participants to think more deeply about applicant 
Intuitions and Reducing Discrimination

qualifications before judgments are made has also been successful, specifically in reducing biases rooted in physical attractiveness (Cann et al., 1981). Highlighting and committing to criteria before decisions are made may then be helpful when judgments are ambiguous.

Reward. A final strategy is a reward for accurate, unbiased behavior. While rewards are not always associated with improved performance (Read, 2005), prior studies have shown that the opportunity to receive a performance-contingent financial incentive may increase motivation or ability to regulate bias and improve overall decision-making (e.g., Lawson et al., 2020; Enke et al., 2021; Stone \& Ziebaryt, 1995; Tosi et al., 1997).

\section{The Present Work}

This work investigated the accuracy of intuitions about reducing socially biased judgment. In Studies 1a-1c, we presented participants with a summary of the JBT and the biases it is known to produce. Participants were then presented with the candidate interventions and judged whether each would reduce discrimination based on physical appearance. In Studies 2a and $2 \mathrm{~b}$, participants were randomly assigned to a control condition or one of the six interventions, allowing us to evaluate the degree to which actual intervention effectiveness aligned with participant intuitions. Results can reveal whether non-experts, who are increasingly responsible for addressing bias in their lives and workplaces, have any insight into what strategies work best for reducing discrimination.

\section{Studies 1a-1c}

\section{Method}

\section{Participants}

In Study 1a, 338 participants recruited via Cloud Research completed the study on Mechanical Turk (42.5\% female, $77.5 \%$ White, $\left.M_{\text {Age }}=37.6, S D=11.6\right)$ and passed two 
attention check items. This sample provided $80 \%$ power to detect an effect size as small as $r$ $=.15$ (see https://osf.io/23q8e/ for Study 1a's pre-registration). In Study 1b, 349 participants from Cloud Research (43.3\% female, $80.8 \%$ White, $\left.M_{\text {Age }}=37.2, S D=11.1\right)$ and in Study $1 \mathrm{c}$, 215 participants from Prolific $\left(66.4 \%\right.$ female, $45.8 \%$ White, $\left.M_{\text {Age }}=25.3, S D=6.6\right)$ completed passed the same two attention check items. See https://osf.io/uxrcs/ for the online supplement as well as data, materials and analysis syntax for all studies. All measures, manipulations, and exclusions are disclosed.

\section{Procedure}

In each study, participants first completed a five-item demographics questionnaire reporting age, gender, race, ethnicity and political orientation. Afterwards, participants read a 150-word summary of the JBT, which was described as a task that required making admissions decisions for a hypothetical academic honor society. Applicants were either objectively more or less qualified, and for each level of qualification applicants were evenly split between more and less physically attractive people. Next, participants viewed three sample JBT applications for five seconds each. Participants were then told about typical JBT performance, which found favoritism towards physically attractive applicants, regardless of qualification (see online supplement for full wording of all instructions). Specifically, participants were told that on average, a physically attractive applicant was about $9 \%$ more likely to be accepted into the honor society despite having the same level of qualifications as a less physically attractive applicant (data calculated from Axt et al., 2018).

Next, participants were told that researchers were about to test six interventions to reduce this favoritism, and it would be their job to review each intervention and predict whether it 
would reduce the bias favoring more physically attractive people. Participants were told that interventions would be administered immediately before people started evaluating applicants.

Afterwards, in a randomized order, participants viewed the materials for each of the six interventions, and were required to spend a minimum of 20 seconds on each page. These interventions included 1) raising accountability (being warned that researchers would review performance to probe for any errors); 2) committing to objectivity (writing down what criteria would be used in evaluation and why those criteria are important); 3) creating implementation intentions to ignore looking at an applicant's face; 4) reviewing a short lesson about confirmation bias; 5) requiring a four-second delay in responses, or 6) rewarding performance by making a $\$ 5$ donation to a charity of a participant's choice if the participant was in the top $10 \%$ of accuracy on the JBT (see online supplement for wording of all interventions).

Participants then completed several items dealing with their perceptions about intervention effectiveness. The first item involved moving the interventions (presented with a short reminder text) into a rank order of overall effectiveness. Next, participants indicated which of the six interventions would reduce discrimination at all. An oversight in the programming of Study 1a required participants to select at least one intervention to be effective, so an option was added in Studies 1b-1c that allowed participants to indicate they believed none of the interventions would be effective. Finally, in Studies 1a-1b, participants completed the rank order item but for how they felt other participants would rank the interventions.

\section{Results}

Table 1 presents the average rank of each intervention and the percentage of participants who believed that each intervention would be effective. In general, results showed considerable variability across participants; for example, the intervention most frequently predicted to be most 
effective was committing to objectivity, but only $24 \%$ of participants held that view in Study 1a, $27.5 \%$ in Study $1 \mathrm{~b}$, and $31.7 \%$ in Study 1c. In addition, there were 58 unique combinations of interventions that were believed to be effective in Study 1a, 54 in Study 1b, and 51 in Study 1c.

Still, a series of Wilcoxon signed-rank tests (used to compare average rankings) revealed consistent patterns between the three samples. In all studies, committing to objectivity was perceived as the most effective intervention (though this did not reliably differ from implementation intentions in Study 1a). Implementation intentions and confirmation bias were either the second or third most effective intervention, and these two did not reliably differ from each other. In Studies 1a-1b, rankings of confirmation bias and accountability did not reliably differ from each other, though in Study 1c accountability was ranked reliably lower. For all studies, delay and accuracy rewards were the lowest-ranked interventions, and only in Study 1c was accuracy reward ranked reliably lower than delay. See the online supplement for results of each test comparing average rankings between interventions.

\section{Discussion}

Overall, participants were confident that several of the intervention strategies would be effective at reducing attractiveness-based discrimination. For example, $61.5 \%$ of participants in Study 1a, $55 \%$ of participants in Study 1b, and 70.6\% of participants in Study 1c thought that three or more interventions would be effective. In all, the three studies showed consistency in what intervention was deemed most effective (committing to objectivity), what interventions were ranked as more moderately effective (reading about confirmation bias or increasing accountability), and what interventions were ranked as the least effective (rewarding accuracy through charitable donations or requiring a delay in responses). 
Intuitions and Reducing Discrimination

In Study $2 \mathrm{a}$ and $2 \mathrm{~b}$, we directly tested how well these predictions aligned with actual effectiveness, by randomly assigning participants to receive one of the six interventions. In particular, we adapted a signal detection theory (Green \& Swets, 1966) approach, and considered an intervention to be successful if, relative to a control condition, the intervention either reduced criterion biases favoring more physically attractive applicants (i.e., lessening the degree to which errors favored more attractive applicants) or increased overall task sensitivity (i.e., reducing the total number of errors made in evaluation), as both of these outcomes have been shown to determine the magnitude of discrimination on the task (Axt \& Lai, 2019).

\section{Study $2 a$ and $2 b$}

\section{Method}

\section{Participants}

In Study 2a, 4011 volunteer participants from the Project Implicit research pool $(64.5 \%$ female, $68.7 \%$ White, $\left.M_{\text {Age }}=35.25, S D=15.40\right)$ completed at least the JBT. Participants were excluded from analysis if they accepted less than $20 \%$ or more than $80 \%$ of applicants, and if they accepted or rejected every more or less physically attractive applicant (Axt et al., 2018), as such behavior suggests participants may have disregarded JBT instructions. This left 3723 eligible participants, which provided greater than $90 \%$ power for detecting a between-subjects

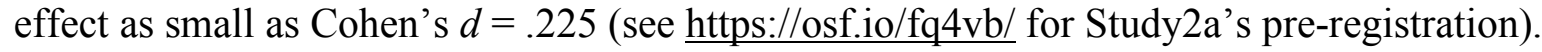

In Study 2b, 2714 participants were recruited from Prolific Academic and were paid $£ 1$ for the completion of the study $\left(39.2 \%\right.$ female, $83.8 \%$ White, $\left.M_{\text {Age }}=26.90, S D=8.96\right)$. Following the same criteria as Study 2a, 145 participant were excluded, which left a sample size with greater than $90 \%$ power for detecting a between subjects effect as small as Cohen's $d=.22$ (given the one-tailed tests specified in Study 2b's pre-registration at https://osf.io/mrty4/). Pre- 
registering one-tailed tests meant we would only reject the null hypothesis if a) within each condition, criterion was lower for more versus less physically attractive applicants (i.e., greater leniency towards more attractive applicants) and b) between conditions, when an intervention either reduced criterion biases favoring more attractive applicants or increased overall sensitivity.

\section{Procedure}

Participants in Study 2a completed four components in the following order: Participants first received the bias-reduction intervention (if there was one), followed by the JBT, a selfreport questionnaire containing measures of perceived performance, desired performance, and explicit attractiveness attitudes, and finally a Brief Implicit Association Test (BIAT) assessing evaluations of more and less physically attractive people. Participants in Study $2 \mathrm{~b}$ completed components in the same order with the exception that there was no BIAT.

Experimental Conditions. Before completing the JBT, participants were randomly assigned to a control condition that received no further instructions or one of the six interventions used in Studies 1a-1c. Participants in the Delay intervention completed a JBT where response options did not appear until four seconds after the application first appeared. In addition, for participants in the Reward condition, a \$5 donation was made to the selected charity for each participant in the top $10 \%$ of overall accuracy.

Judgment Bias Task. Participants completed a 64-trial physical attractiveness JBT that was the same as those from prior research (e.g., Axt \& Lai, 2019; Axt \& Johnson, 2021). Each applicant profile contained four pieces of relevant information: Science GPA (on a scale of 1-4), Humanities GPA (on a scale of 1-4), letter of recommendation quality (with four categories:

poor, fair, good, excellent), and interview score (on a scale of 1-100). Participants were asked to accept approximately half of the applicants. Profiles were constructed such that half were more 
qualified and half were less qualified. Qualification was calculated by converting each piece of information to a 1 to 4 scale (GPAs retained their four-point scale, interview scores were divided by 25 , and recommendation letter quality was converted such that Poor $=1$, Fair $=2$, Good $=3$ and Excellent $=4$ ). More qualified applicants had a score of 14 and less qualified applicants had a score of 13 . Within each level of qualification, there were an equal number of male and female White faces that were pre-rated to be more versus less physically attractive $(d=2.64$ in Axt et al., 2018). Only White stimuli were used to remove the possible influence of race on participants' judgment, though this decision reduces the generalizability of results.

Each participant was randomly assigned to one of twelve possible JBT orders; across orders, each face was equally likely to be paired with a more versus less qualified profile.

Self-Report Measures. Participants completed three self-report items measuring perceived performance, desired performance, and explicit attractiveness preferences, each using a seven-point scale. For perceived performance, participants chose -3 when they were "extremely easier on less physically attractive applicants and tougher on more physically attractive applicants," +3 when they were "extremely easier on more physically attractive applicants and tougher on less physically attractive applicants", and a midpoint of 0 for "I treated both physically unattractive and physically attractive applicants equally". The desired performance measure used the same structure but investigated how participants wanted to perform. Finally, for explicit attitudes, participants chose -3 when they "strongly prefer physically unattractive people to physically attractive people," +3 when they "strongly prefer physically unattractive people to physically attractive people", and a midpoint of 0 when they "like physically attractive and physically unattractive people equally". See online supplement for full wording. 
Intuitions and Reducing Discrimination

Across studies, a large majority of participants reported a perception of having been unbiased (82.9\% in Study 2a, 76.9\% in Study 2 b) and a desire to be unbiased $(93.7 \%$ in Study 2a, $85.9 \%$ in Study 2b), though explicit attitudes still showed relatively large preferences for physically attractive people $(d=.73$ in Study $2 \mathrm{a}, d=.77$ in Study $2 \mathrm{~b})$.

\section{Brief Implicit Association Test}

Participants in Study 1a completed a four-block, good-focal Brief Implicit Association Test (BIAT; Sriram \& Greenwald, 2009) which measured evaluations toward more versus less physically attractive people. Stimuli for each attractiveness group were two male and two female faces pre-selected from the same images used in the JBT. Responses were analyzed using the $D$ scoring algorithm (Nosek et al., 2014), with a higher score indicating more positive implicit associations toward more versus less physically attractive people (see online supplement for instructions). Study la participants showed a strong effect of more positive associations towards more physically attractive people (Cohen's $d=1.39$ ).

\section{Results}

In both studies, accuracy on the JBT (accepting more qualified and rejecting less qualified applicants) was above chance performance of 50\% (Study 2a: $M=67.8 \%, S D=8.5$; Study 2b: $M=66.9 \%, S D=8.8$ ), and levels of sensitivity were above zero (Study $2 \mathrm{a}: M=1.04$, $S D=.55$; Study $2 \mathrm{~b}: M=.96, S D=.54)$. The average acceptance rate was also close to the recommended $50 \%$ (Study $2 \mathrm{a}=51.5 \%$; Study $2 \mathrm{~b}=52.4 \%)$.

We first conducted a series of paired samples $t$-test in each condition comparing the criterion for more versus less physically attractive applicants to investigate the presence of a response bias favoring more physically attractive applicants. In Study 2a, all conditions showed a lower criterion for more physically attractive applicants, except for the Implementation 
Intentions conditions, which did not show any evidence of a criterion bias $(d=.04)$. In Study $2 \mathrm{~b}$, criterion for physically attractive applicants was significantly lower than that for less physically attractive applicants in all conditions. See Table 2 for descriptive statistics of overall JBT accuracy, sensitivity, and criterion, and see Table 3 for results of paired-samples $t$-tests for criterion values within each condition.

Next, we conducted a series of independent samples $t$-tests comparing the control condition to each experimental condition, both for overall sensitivity and for level of criterion bias (calculated as a difference score between the two values, such that higher scores indicate greater leniency towards more versus less physically attractive applicants). In Study 2a, criterion bias was only reduced in the Implementations Intentions condition $(d=-.23)$, and sensitivity was only increased in the Delay condition $(d=.22)$. Similar results were found in Study $2 \mathrm{~b}$, where only the Implementation Intentions condition reduced criterion bias $(d=-.19)$ and only the Delay condition increased sensitivity $(d=.33)$. See Table 4 for test statistics.

The online supplement has descriptive statistics for measures of implicit and explicit attitudes as well as for perceived and desired performance. A series of exploratory analyses, reported in the online supplement, compared whether any intervention changed perceived performance, desired performance, or explicit attitudes. No consistent results were found across studies. In Study 2a, relative to the control condition, both the confirmation bias $(d=.19)$ and Delay intervention $(d=.13)$ conditions were associated with increased BIAT $D$ scores, showing more positive associations with physically attractive people.

\section{Evaluating the accuracy of participant intuitions.}

There are several possible methods for evaluating the accuracy of predictions made by Study 1a-1c participants concerning the effectiveness of each intervention. The most stringent 
method would be to look at the number of participants who were "perfectly right". Only $0.6 \%$ of participants in Study 1a, 0.9\% of participants in Study 1b, and 0\% of participants in Study 1c correctly guessed that only the implementation intentions and delay interventions would reduce discrimination; if anything, these rates were lower than the percentage of participants who were "perfectly wrong" in thinking that only those two interventions would fail to reduce discrimination (Study $1 \mathrm{a}=2.1 \%$, Study $1 \mathrm{~b}=2.3 \%$, Study $1 \mathrm{c}=2.8 \%$ ).

A second approach involves investigating accuracy for each individual intervention against chance responding (i.e., whether participants showed greater or worse accuracy than a chance responding rate of 50\%). Here, a series of one-proportion $Z$ tests (see online supplement for analyses) found that, across studies, participants were above chance in predicting the (lack of) effectiveness for the reward manipulation and were above chance in predicting the actual effectiveness of the implementation intentions interventions; at the same time, participants were below chance in predictions about the effectiveness of the commitment to objectivity, delay and confirmation bias interventions.

A final approach uses the rank ordering of interventions through a profile correlation analysis (Rogers et al., 2018). In this context, a profile correlation analysis can assess the degree of agreement between a participant's ranking of intervention effectiveness and actual intervention effectiveness. To complete this analysis, data from Studies $2 \mathrm{a}-2 \mathrm{~b}$ were used to rank each intervention by first ranking those interventions that reliably changed either criterion bias or sensitivity (prioritizing that which produced the largest effect) and then, among interventions that did not reliably change criterion bias or sensitivity, ranking interventions by the weighted average across studies for the overall impact on criterion bias or sensitivity. This procedure produced the following ranking: 1) Delay, 2) Implementation Intentions, 3) Confirmation Bias, 
4) Objectivity, 5) Accuracy Reward, 6) Accountability. Profile correlation analyses found that in Study $1 \mathrm{a}(q=-.02)$, Study $1 \mathrm{~b}(q=-.02)$ and Study $1 \mathrm{c}(q=.08)$, the average profile correlation between each participant's rankings and overall intervention effectiveness was essentially zero (see online supplement for further reporting). That is, there was no evidence that participants on average produced rankings of anticipated intervention effectiveness that were reliably correlated with rankings of actual effectiveness.

\section{Discussion}

In a comparative test of six intervention strategies, two were consistently effective across studies; creating an implementation intention to avoid using faces reduced discrimination through lessening relative biases in criterion and, in a replication of prior work (Axt \& Lai, 2019), requiring a delay in responding lessened discrimination through reducing the total number

of errors made on the JBT. Despite the high statistical power to detect even small effects, none of the other four intervention strategies impacted JBT performance. Across several analytic approaches, there was also little evidence that participants had any insight into the collective effectiveness or ineffectiveness of these interventions.

\section{General Discussion}

In three studies, participants reviewed six interventions meant to reduce discrimination favoring physically attractive people in a judgment task. Responses were characterized by a fair amount of confidence in at least some interventions - across studies, $61 \%$ of participants thought that at least half of the interventions would reduce discrimination - as well as by large variability in what specific interventions would work - even the most common combination of responses was only shared among 10\% of participants in Study 1a, 6.9\% of participants in Study 1b, and $13.4 \%$ of participants in Studylc. 
However, in two high-powered tests of the effectiveness of these interventions, participants' intuitions were not well-aligned with actual results. In both samples, relative to a Control condition, the Delay intervention increased overall sensitivity and the Implementation Intentions intervention reduced biases in response criterion, with the only inconsistency across samples being that the latter intervention fully debiased behavior in Study 1a but not Study $1 \mathrm{~b}$. Fewer than $1 \%$ of participants correctly identified the two of six interventions that actually changed JBT performance, and when evaluating each intervention individually, participants were more likely to be below than above chance in guessing whether or not each intervention would impact the magnitude of attractiveness-based discrimination. In short, participants lacked any substantive insight into how well this collection of intervention strategies would perform when deployed on novel participants.

This research speaks to ongoing efforts to address discrimination in real-life situations like hiring, promotion, and admissions. Many non-experts are facing renewed efforts to address socially-biased discrimination within their organization, a population that has shown a continued reliance on intuition when evaluating others (e.g., Dana et al., 2013; Highhouse, 2008; Hoffman et al., 2018; Miles \& Sadler-Smith, 2014). Here, we extend this work to the question of accuracy of intuitions concerning how to reduce discrimination within the specific context of physical attractiveness, and while it remains possible for intuitions to be more accurate in other domains, the present results do not provide much confidence on individuals' ability to meaningfully address discrimination when relying solely on intuition.

Practically, our results suggest that decisions made to address biases will be ineffective when not based in evidence. As a result, the data presented here can hopefully be used to convince decision-makers that it is important to look beyond one's gut when thinking about bias 
reduction. Similarly, the studies also speak to a greater urgency among researchers to test interventions in more naturalistic field settings and to prioritize engaging with real-world practitioners who may be able to implement such findings.

Theoretically, these results add nuance to models of biased judgment (Fazio, 1990; Wilson \& Brekke, 1994) by expanding prior work to now illustrate that while people can often lack insight into the social information driving their biased judgment, they also lack insight into what changes will effectively limit its influence. One implication of these results is that some participants in Studies 2a-2b may have indeed realized that their judgments favored more physically attractive applicants, and as a result deployed a counteractive strategy based on their own intuition to correct for this influence. However, the inaccuracy in predictions from Studies 1a-1c indicates that many intervention strategies that people believe to be effective do not actually change behavior, at least in this context. As a result, discrimination on a task like the JBT may not only be fueled by those who fail to notice the influence of biasing information (here, physical attractiveness) but also by those who are aware of the biasing information but lack insight into what psychological processes need to occur in order to reduce its influence. Alerting people to their biased behavior may then be insufficient for creating change; it appears to be just as important to provide effective strategies for combatting bias.

\section{Implications for Efforts to Reduce Discrimination}

While the focus of this work is primarily on the alignment between participant intuitions and actual intervention effectiveness, Studies $2 \mathrm{a}-2 \mathrm{~b}$ also provide the largest comparative test of several prominent bias-reduction strategies using the same outcome measure. Despite developing six interventions based on prior academic work, only two were consistently successful at changing JBT performance; an implementation intentions strategy reduced criterion biases 
favoring more physically attractive applicants, and the delay intervention decreased the number of errors in which such favoritism could occur (i.e., through increased sensitivity). Though we lack definitive evidence behind why these interventions are effective, past work suggests that it is likely that participants in the Delay intervention used the extra time to better parse decisionrelevant information like GPA or interview score, thereby raising the threshold for how much information needed to be gathered before a decision could be reached (Axt \& Johnson, 2021). Curiously, participants in the Implementation Intentions condition were told to ignore applicant faces, yet results found reductions in criterion biases but no changes in overall sensitivity. Given past work, these participants then looked more like those warned to avoid using physical attractiveness in their decisions, who showed reductions in criterion bias (Axt \& Johnson, 2021), and less like participants who completed a blinded version of the JBT where applicant faces were removed entirely, who showed increases in overall sensitivity (Axt \& Lai, 2019). This pattern suggests that when being warned to ignore the face, participants in the Implementation Intention condition may have instead actually paid greater attention to applicant faces. As participants noticed that applicants varied on attractiveness, they may have worked to combat this bias by being more lenient towards less attractive applicants and more stringent towards more attractive applicants, resulting in no changes in the number of errors made (and therefore no differences in overall sensitivity). The Implementation Intentions intervention may have then been effective because it guided participants to attend to and then counteract the influence of physical attractiveness rather than ignoring attractiveness entirely, results that are consistent with other uses of this strategy, such as in reducing bias on measures of implicit associations (Lai et al., 2014; Webb et al. 2012). 
However, the present studies should not be taken as the final word on the relative effectiveness of any of these interventions. Aside from being constrained to a single outcome measure, many interventions may have overlooked crucial components that could have impacted performance. For instance, in the Accountability condition, participants were only warned that their behavior would be evaluated and analyzed by a group of experts. This approach may have failed to adequately heighten accountability, as prior studies have also included the possibility of participants having to justify their decisions to others (Kennedy, 1993). Similarly, in the confirmation bias manipulation, participants only reviewed a 236-word explanation of confirmation bias and a read about a potential strategy for how to reduce it. One plausible reason for the ineffectiveness of the intervention was that this manipulation did not provide enough practice at combatting confirmation bias. In a prior study (Sellier et al., 2019), participants received multiple interventions to reduce confirmation bias, including a series of exercises eliciting confirmation bias and correction. Given past research on the "bias blind spot" (Pronin et al., 2002), it is possible that merely passively absorbing information about confirmation bias is insufficient to change behavior.

Finally, in the Reward condition, participants selected a charity that would receive a $\$ 5$ donation if their performance on the JBT were in the top $10 \%$ based on JBT accuracy. This more distant type of reward may then not have created enough of an incentive for participants to invest in greater cognitive resources to inhibit bias and improve accuracy. Previous work demonstrating the causal relationship between performance-contingent financial incentive and better decisionmaking (Stone \& Ziebert, 1995; Tosi et al., 1997) all used direct payment to participants. Research on reducing discrimination will benefit from investigating whether different operationalizations of these intervention strategies will be more successful in changing behavior, 
either on the JBT or other outcomes, and doing so may in turn highlight the essential components needed for each intervention to be effective (e.g., Axt, Casola \& Nosek, 2019).

Alternatively, the interventions used here may have been faithful versions of each strategy, but these approaches simply do not extend to attractiveness-based discrimination. It is possible that biases based on physical attractiveness are more subtle and resistant to change than biases based on more salient social dimensions. Relatedly, a large majority of participants (80.7\% across all conditions in Studies $2 \mathrm{a}-2 \mathrm{~b})$ reported not thinking they used physical attractiveness in their judgments, meaning that even if participants were committed to being objective, any enhanced objectivity may have been ineffective at changing behavior if they did not realize the influence of attractiveness on their judgments (e.g., Uhlmann \& Cohen, 2007). This perspective may explain why only the Implementation Intentions intervention effectively reduced biases in criterion, as it was the only intervention that explicitly named the source of the biasing social information.

In all, our studies tell us much more about the accuracy of participant intuitions when evaluating specific forms of bias-reducing interventions in a single context, and tell us much less about the overall validity of these larger intervention strategies. However, the inability to parse whether intervention effectiveness stems from an unsuccessful operationalization versus the context of physical attractiveness does not detract from our conclusions regarding the general inaccuracy of participant intuitions. All participants in Studies 1a-1c were exposed to the exact materials that were then used in Studies $2 \mathrm{a}-2 \mathrm{~b}$. While additional tests of different versions of these interventions and across different forms of social biases will be useful, at minimum the present work indicates that more basic information is needed for practitioners and researchers 
concerning 1) the specific characteristics that are necessary to render these intervention strategies effective and 2) the judgment contexts in which they can be applied.

\section{Limitations}

One potential criticism for this work concerns the exclusive focus on physical attractiveness, which while impactful may not be the type of discrimination that many participants think about often. It is then unclear whether the intuitions measured here would function similarly when applied to other, more salient forms of discrimination, such as pertaining to age, gender, or race. To investigate this issue, we ran a follow-up study looking at the degree to which people thought these interventions would function similarly across different social dimensions. Specifically, participants $(N=157)$ reviewed the JBT and five of the interventions used in Studies $1 \mathrm{a}-1 \mathrm{c}^{1}$, first reporting whether each would be effective at removing bias based on physical attractiveness, and then indicating whether they believed each intervention would have the same impact when the JBT was adapted to measure biases related to either age, race, or gender (see online supplement for materials). Across all interventions, $77.7 \%$ of participants indicated that they believed each intervention would have the same impact on physical attractiveness biases as on biases related to age, gender, or race (minimum $=72.0 \%$ for the delay intervention, maximum $=82.2 \%$ for the accountability intervention).

These results indicate that for a large majority of participants, the interventions studied here are thought to work similarly when applied to more salient forms of discrimination. At the same time, though more than $85 \%$ of Study $2 \mathrm{a}-2 \mathrm{~b}$ participants reported not wanting to use physical attractiveness in their judgments, it is possible that these interventions may have

\footnotetext{
${ }^{1}$ We removed the implementation intentions intervention since the manipulation specifically mentions applicant faces, and as a result may have confused participants when asked whether it would generalize to other forms of social biases.
} 
produced different outcomes when applied to forms of discrimination where participants likely have even higher vigilance against appearing biased, such as for race or gender.

Another limitation may stem from our treatment of the delay intervention. Participants may not have considered lessening noise (that is, improving overall task accuracy) as a form of discrimination reduction (Axt \& Lai, 2019). Rather, participants in Studies 1a-1c might have thought about changes to performance that were specific to how more versus less physically attractive people were evaluated. This is a fair critique, and one that arose due to a decision to present more straightforward instructions to participants (i.e., not having to explain the distinction between bias and noise). However, even when disregarding the delay intervention, results do not instill much confidence in the accuracy of intuition. When excluding the delay condition, $5.8 \%$ of participants across studies would have been "perfectly right" in predicting how interventions would fare at reducing discrimination, compared to $2.8 \%$ of participants that would have been "perfectly wrong". In addition, the one-proportion $Z$ tests reported earlier would have still found just as many interventions to be above chance as below chance in accuracy when predicting the effectiveness of the five other interventions. Finally, even when only ordering all six interventions based solely on their effect on criterion biases, profile correlations showed no strong association between participant rankings and actual rankings (Study 1a $q=.04$, Study $1 \mathrm{~b} q=.04$, Study $1 \mathrm{c} q=.13$ ).

A final limitation is differences among sample sources, as the current studies used data from Prolific, Mechanical Turk, and Project Implicit. These differences in samples may have complicated participants' ability to predict how various interventions would fare. However, as reported in the online supplement, even when only looking at our two Prolific samples (Study 1c and Study 2b), participants did not show much accuracy in their predictions regarding 
intervention effectiveness. For instance, one-sample Z-tests still found that Prolific participants were more likely to be below than above chance in predicting the effectiveness of each intervention on other Prolific participants. In addition, when ranking interventions based only on Study $2 \mathrm{~b}$ results, profile correlations still showed no strong association between predicted and actual intervention effectiveness $(q=.10)$. While these analyses suggest that the observed inaccuracy in intuition is not merely a function of mismatches between sample sources, it remains possible that predictions would be considerably more accurate if participants were to come from the same organization or affinity group.

While two of the interventions were effective at reducing discrimination, the durability of this effectiveness is currently unknown, and prior work focusing on implicit associations has found that manipulations that changed performance immediately did not persist for even twentyfour hours (Lai et al., 2016). Understanding the durability of the implementation intentions intervention used here will be useful, especially in informing related work concerning long-term changes in prejudice (e.g., Forscher et al., 2017). Finally, as mentioned earlier, the present work was constrained to one outcome measure, one type of judgment, one form of social bias, and one operationalization of each intervention strategy. While this narrow focus does not impact our specific conclusions regarding the accuracy of participant intuitions in this context, it is possible that such accuracy could change when using other measures, when applied to other types of bias, and when tested across other versions of these interventions. Testing the generalizability of these findings is a clear avenue for subsequent work.

\section{Future Directions}

This research program may benefit from further exploration of two related questions.

First, the present studies did not collect any information about participant confidence in Studies 
1a-1c. It is unclear whether the predictions made about intervention effectiveness were highly confident (and largely inaccurate) or were merely reflecting participants" "best guess", meaning participants would not have then been overly surprised to learn about how often they were wrong in their responses. Prior work on more factual judgments suggests that participants are typically overconfident in their predictions, but this overconfidence can vary substantially across domains (e.g., Soll \& Klayman, 2004), and the role of overconfidence in predictions about bias-reducing interventions is currently unknown. Greater knowledge of the role participant confidence will also shed light on the real-world implications of this issue.

Second, these samples were limited to individuals without expertise in research on biased judgment. Prior work has found that experts have some accuracy in predicting the outcomes of both replication studies (Dreber et al., 2015; Forsell et al., 2019) and novel data collections (DellaVigna \& Pope, 2018), and the same may be true of experts when predicting the relative success of the interventions used here, though the fact that each of these interventions was based on the success of prior work may lead even experts to suffer from the same levels of inaccuracy as exhibited by non-expert participants. Regardless, an expert sample, of either researchers familiar with these strategies or professionals with significant hiring or admissions experience, will be informative for understanding whether insight into the effectiveness of interventions can be developed over time. Similarly, it is possible that participants who were more likely to have been the target of discrimination, either through identifying as being low in physical attractiveness or through having another stigmatized identity, may have greater insight into which of these interventions would most effectively reduce discrimination on the JBT. 
Intuitions and Reducing Discrimination

\section{Conclusion}

Increased calls to address discrimination within organizations will lead to the implementation of new interventions and changes, a push that is complicated by a general lack of evidence-based methods for addressing bias and by a widespread faith in intuition when evaluating others. Results of two studies testing six different manipulations found that a pair of interventions -- rehearsing distraction-inhibiting if-then statements and requiring a delay in responses -- significantly reduced the magnitude of attractiveness-based discrimination on a judgment task, though these results were poorly aligned with predictions about how to address such discrimination.

Aside from being the largest comparative assessment to date of strategies to reduce biased judgment, this work reveals that, just as people can often have little insight into the social information influencing their behavior, this lack of insight extends into strategies for minimizing biased behavior. This work shows that addressing bias will take more than good intentions and strong feelings, and results only further stresses the need for the creation and adoption of effective, evidence-based practices in reducing discrimination. 
Intuitions and Reducing Discrimination

\section{References}

Amodio, D. M., Master, S. L., Yee, C. M., \& Taylor, S. E. (2007). Neurocognitive components of the behavioral inhibition and activation systems: Implications for theories of selfregulation. Psychophysiology, 45, 11-19.

Ariely, D., \& Zakay, D. (2001). A timely account of the role of duration in decision making. Acta Psychologica, 108, 187-207.

Axt, J.R., Casola, G.M. \& Nosek, B.A (2019). Reducing social judgment biases may require identifying the potential source of bias. Personality and Social Psychology Bulletin, 45, $1232-1251$

Axt, J. R., Nguyen, H., \& Nosek, B. A. (2018). The Judgment Bias Task: A flexible method for assessing individual differences in social judgment biases. Journal of Experimental Social Psychology, 76, 337-355.

Axt, J.R. \& Johnson, D.J. (2021). Understanding mechanisms behind discrimination using diffusion decision modeling. Journal of Experimental Social Psychology. Advance online publication.

Axt, J.R. \& Lai, C.K. (2019). Reducing discrimination: A bias versus noise perspective. Journal of Personality and Social Psychology, 117, 26-49.

Cann, A., Siegfried, W. D., \& Pearce, L. (1981). Forced attention to specific applicant qualifications: Impact on physical attractiveness and sex of applicant biases. Personnel Psychology, 34, 65-75.

Chandler, J. J., \& Pronin, E. (2012). Fast thought speed induces risk taking. Psychological Science, 23, 370-374.

Chang, E. H., Milkman, K. L., Gromet, D. M., Rebele, R. W., Massey, C., Duckworth, A. L., \& 
Intuitions and Reducing Discrimination

Grant, A. M. (2019). The mixed effects of online diversity training. Proceedings of the National Academy of Sciences, 116, 7778-7783.

Commisso, M., \& Finkelstein, L. (2012). Physical attractiveness bias in employee termination. Journal of Applied Social Psychology, 42, 2968-2987

Crosby, F., Bromley, S., \& Saxe, L. (1980). Recent unobtrusive studies of Black and White discrimination and prejudice: A literature review. Psychological Bulletin, 87, 546563.

Dana, J., Dawes, R., \& Peterson, N. (2013). Belief in the unstructured interview: The persistence of an illusion. Judgment and Decision Making, 8, 512-520.

DellaVigna, S., \& Pope, D. (2018). What motivates effort? Evidence and expert forecasts. The Review of Economic Studies, 85, 1029-1069.

Dobbin, F., Kalev, A., \& Kelly, E. (2007). Diversity management in corporate America. Contexts, 6, 21-27.

Dovidio, J. F., \& Gaertner, S. L. (2000). Aversive racism and selection decisions: 1989 and 1999. Psychological Science, 11, 315-319.

Dovidio, J.F., Hewstone, M., Glick, P. \& Esses, V. (2010). Prejudice, stereotyping, and discrimination: Theoretical and empirical overview. In J. F. Dovidio, M. Hewstone, P. Glick \& V. M. Esses (Eds.), The Sage Handbook of Prejudice, Stereotyping, and Discrimination (pp. 97-114). Los Angeles, CA: Sage

Downs, A. C., \& Lyons, P. M. (1991). Natural observations of the links between attractiveness and initial legal judgments. Personality and Social Psychology Bulletin, 17, 541-547.

Dreber, A., Pfeiffer, T., Almenberg, J., Isaksson, S., Wilson, B., Chen, Y., ... \& Johannesson, M. 
Intuitions and Reducing Discrimination

(2015). Using prediction markets to estimate the reproducibility of scientific research. Proceedings of the National Academy of Sciences, 112, 15343-15347.

Enke, B., Gneezy, U., Hall, B., Martin, D., Nelidov, V., Offerman, T., \& van de Ven, J. (2020). Cognitive biases: Mistakes or missing stakes? CESifo Working Paper Series, 8168.

Fazio, R. H. (1990). Multiple processes by which attitudes guide behavior: The MODE model as an integrative framework. Advances in Experimental Social Psychology, 23, 75-109

Fisher, P., Risavy, S., Robie, C., Konig, C., Christiansen, N., Tett, R., \& Simonet, D. (2020). Selection myths: A conceptual replication of HR professionals' beliefs about effective human resource practices in the United States and Canada. Journal of Personnel Psychology, 20, 51-60.

Fiske, S. T. (1998). Stereotyping, prejudice, and discrimination. In D. T. Gilbert, S. T. Fiske, \& G. Lindzey (Eds.), The handbook of social psychology (p. 357-411). McGraw-Hill.

Forscher, P. S., Mitamura, C., Dix, E. L., Cox, W. T., \& Devine, P. G. (2017). Breaking the prejudice habit: Mechanisms, timecourse, and longevity. Journal of Experimental Social Psychology, 72, 133-146.

Forsell, E., Viganola, D., Pfeiffer, T., Almenberg, J., Wilson, B., Chen, Y., ... \& Dreber, A. (2019). Predicting replication outcomes in the Many Labs 2 study. Journal of Economic Psychology, 75, Article 102117.

Goldin, C., \& Rouse, C. (2000). Orchestrating impartiality: The impact of "blind" auditions on female musicians. American Economic Review, 90, 715-741.

Green, D. M., \& Swets, J. A. (1966). Signal detection theory and psycho-physics. New York: Wiley. (Reprinted 1974, Huntington, NY: Krieger).

Hale, G., Regev, T., \& Rubinstein, Y. (2021). Do looks matter for an academic career in 
Intuitions and Reducing Discrimination

economics? Center for Economic Policy Research. Retrieved from: https://papers.ssrn.com/sol3/papers.cfm?abstract_id=3805308

Highhouse, S. (2008). Stubborn reliance on intuition and subjectivity in employee selection. Industrial and Organizational Psychology, 1, 333-342.

Hoffman, M., Kahn, L. B., \& Li, D. (2018). Discretion in hiring. The Quarterly Journal of Economics, 133, 765-800.

Hosoda, M., Stone-Romero, E. F., \& Coats, G. (2003). The effects of physical attractiveness on job-related outcomes: A meta-analysis of experimental studies. Personnel Psychology, $56,431-462$.

Jaeger, B., Todorov, A., Evans, A., \& van Beest, I. (2019). Can we reduce facial biases? Persistent effects of facial trustworthiness on sentencing decisions. Journal of Experimental Social Psychology, 90, 104004.

Kenealy, P., Gleeson, K., Frude, N., \& Shaw, W. (1991). The importance of the individual in the 'causal'relationship between attractiveness and self-esteem. Journal of Community \& Applied Social Psychology, 1(1), 45-56.

Kennedy, J. (1993). Debiasing audit judgment with accountability: A framework and experimental results. Journal of Accounting Research, 31, 231-245.

Lai, C. K., Marini, M., Lehr, S. A., Cerruti, C., Shin, J. E. L., Joy-Gaba, J. A., ... \& Nosek, B. A. (2014). Reducing implicit racial preferences: I. A comparative investigation of 17 interventions. Journal of Experimental Psychology: General, 143(4), 1765-1785.

Lai, C. K., Skinner, A. L., Cooley, E., Murrar, S., Brauer, M., Devos, T., ... \& Nosek, B. A. (2016). Reducing implicit racial preferences: II. Intervention effectiveness across time. Journal of Experimental Psychology: General, 145, 1001-1016. 
Intuitions and Reducing Discrimination

Lawson, M. A., Larrick, R. P., \& Soll, J. B. (2020). Comparing fast thinking and slow thinking: The relative benefits of interventions, individual differences, and inferential rules. Judgment and Decision Making, 15(5), 660-684.

Lerner, J. S., \& Tetlock, P. E. (1999). Accounting for the effects of accountability. Psychological Bulletin, 125, 255-275.

Maestripieri, D., Henry, A., \& Nickels, N. (2017). Explaining financial and prosocial biases in favor of attractive people: Interdisciplinary perspectives from economics, social psychology, and evolutionary psychology. Behavioral and Brain Sciences, 40, E19.

Marlowe, C. M., Schneider, S. L., \& Nelson, C. E. (1996). Gender and attractiveness biases in hiring decisions: Are more experienced managers less biased?. Journal of Applied Psychology, 81, 11-21.

McDaniel, M. A., Whetzel, D. L., \& Schmidt, F. L. (1994). The validity of employment interviews: a comprehensive review and meta-analysis. Journal of Applied Psychology, 79, 599-616.

Mendoza, S. A., Gollwitzer, P. M., \& Amodio, D. M. (2010). Reducing the expression of implicit stereotypes: Reflexive control through implementation intentions. Personality and Social Psychology Bulletin, 36, 512-523

Miles, A., \& Sadler-Smith, E. (2014). " With recruitment I always feel I need to listen to my gut": The role of intuition in employee selection. Personnel Review, 43, 606-627.

Moss-Racusin, C. A., Dovidio, J. F., Brescoll, V. L., Graham, M. J., \& Handelsman, J. (2012). Science faculty's subtle gender biases favor male students. Proceedings of the National Academy of Sciences of the United States of America, 109, 16474-16479.

Nisbett, R. E., \& Wilson, T. D. (1977). Telling more than we can know: Verbal reports on 
Intuitions and Reducing Discrimination

mental processes. Psychological Review, 84, 231-259.

Nosek, B. A., Bar-Anan, Y., Sriram, N., Axt, J.R., \& Greenwald, A. G. (2014). Understanding and using the Brief Implicit Association Test: Recommended scoring procedures. PloS One, 9, e110938.

Palmer, J. K., \& Feldman, J. M. (2005). Accountability and need for cognition effects on contrast, halo, and accuracy in performance ratings. The Journal of Psychology, 139, 119-138.

Paolini, S., Crisp, R. J., \& McIntyre, K. (2009). Accountability moderates member-to-group generalization: Testing a dual process model of stereotype change. Journal of Experimental Social Psychology, 45, 676-685.

Plant, E. A., \& Devine, P. G. (1998). Internal and external motivation to respond without prejudice. Journal of Personality and Social Psychology, 75, 811-832.

Pronin, E., Lin, D. Y., \& Ross, L. (2002). The bias blind spot: Perceptions of bias in self versus others. Personality and Social Psychology Bulletin, 28, 369-381.

Read, D. (2005). Monetary incentives, what are they good for? Journal of Economic Methodology, 12, 265-276.

Recruitics (2021, September 15). LinkedIn creates features aimed to reduce bias. Recruitment Marketing Blog. Retrieved from https://info.recruitics.com/blog/linkedin-creates-featureaimed-to-reduce-bias.

Rogers, K. H., Wood, D., \& Furr, R. M. (2018). Assessment of similarity and self-other agreement in dyadic relationships: A guide to best practices. Journal of Social and Personal Relationships, 35, 112-134.

Schmidt, F. L., \& Hunter, J. E. (1998). The validity and utility of selection methods in personnel 
Intuitions and Reducing Discrimination

psychology: Practical and theoretical implications of 85 years of research findings. Psychological Bulletin, 124, 262-274.

Shankland, S. (2021, August 9). Twitter AI bias contest shows beauty filters hoodwink the algorithm. CNet. Retrieved from https://www.cnet.com/tech/mobile/twitter-ai-biascontest-shows-beauty-filters-hoodwink-the-algorithm/

Simner, M. L., \& Goffin, R. D. (2003). A position statement by the International Graphonomics Society on the use of graphology in personnel selection testing. International Journal of Testing, 3, 353-364.

Soll, J. B., \& Klayman, J. (2004). Overconfidence in interval estimates. Journal of Experimental Psychology: Learning, Memory, and Cognition, 30, 299-314.

Sellier, A. L., Scopelliti, I., \& Morewedge, C. K. (2019). Debiasing training improves decision making in the field. Psychological Science, 30, 1371-1379.

Stone, D. N., \& Ziebart, D. A. (1995). A model of financial incentive effects in decision making. Organizational Behavior and Human Decision Processes, 61, 250-261.

Tosi, H. L., Katz, J. P., \& Gomez-Mejia, L. R. (1997). Disaggregating the agency contract: The effects of monitoring, incentive alignment, and term in office on agent decision making. Academy of Management Journal, 40, 584-602.

Uhlmann, E. L., \& Cohen, G. L. (2005). Constructed criteria: Redefining merit to justify discrimination. Psychological Science, 16, 474-480.

Uhlmann, E. L., \& Cohen, G. L. (2007). “I think it, therefore it's true”: Effects of self-perceived objectivity on hiring discrimination. Organizational Behavior and Human Decision Processes, 104, 207-223.

Van der Zee, K. I., Bakker, A. B., \& Bakker, P. (2002). Why are structured interviews so rarely 
Intuitions and Reducing Discrimination

used in personnel selection?. Journal of Applied Psychology, 87, 176-184.

Vieider, F. M. (2009). The effect of accountability on loss aversion. Acta Psychologica, 132, 96101.

Webb, T. L., Sheeran, P., \& Pepper, J. (2012). Gaining control over responses to implicit attitude tests: Implementation intentions engender fast responses on attitude-incongruent trials. British Journal of Social Psychology, 51, 13-32.

Wilson, T. D., \& Brekke, N. (1994). Mental contamination and mental correction: Unwanted influences on judgments and evaluations. Psychological Bulletin, 116, 117-142. 
Intuitions and Reducing Discrimination

Table 1. Average rankings and percentage selected as effective for each intervention.

\begin{tabular}{|c|c|c|c|c|c|c|}
\hline & \multicolumn{2}{|c|}{ Study 1a } & \multicolumn{2}{|c|}{ Study $1 b$} & \multicolumn{2}{|c|}{ Study 1c } \\
\hline Intervention & Average Rank & $\%$ Effective & Average Rank & $\%$ Effective & Average Rank & $\%$ Effective \\
\hline Accountability & $3.34^{\mathrm{c}}$ & $47.0 \%$ & $3.42^{\mathrm{c}}$ & $47.9 \%$ & $3.40^{\mathrm{c}}$ & $48.6 \%$ \\
\hline Objectivity & $2.84^{\mathrm{a}}$ & $63.3 \%$ & $2.66^{\mathrm{a}}$ & $60.7 \%$ & $2.49^{\mathrm{a}}$ & $79.9 \%$ \\
\hline Implementation Intentions & $3.04^{\mathrm{a}, \mathrm{b}}$ & $63.3 \%$ & $3.13^{b}$ & $52.1 \%$ & $3.18^{b, c}$ & $59.8 \%$ \\
\hline Confirmation Bias & $3.20^{\mathrm{b}, \mathrm{c}}$ & $58.6 \%$ & $3.23^{b, c}$ & $49.9 \%$ & $2.98^{b}$ & $60.7 \%$ \\
\hline Delay & $4.24^{\mathrm{d}}$ & $28.1 \%$ & $4.24^{\mathrm{d}}$ & $26.1 \%$ & $4.13^{\mathrm{d}}$ & $36.0 \%$ \\
\hline Reward & $4.33^{\mathrm{d}}$ & $24.9 \%$ & $4.33^{d}$ & $30.7 \%$ & $4.81^{\mathrm{e}}$ & $22.9 \%$ \\
\hline
\end{tabular}

Note: Interventions sharing a superscript letter did not reliably differ from each other (see online supplement for results of each test). 
Intuitions and Reducing Discrimination

Table 2. Means (and Standard Deviations) for Overall JBT Accuracy, Sensitivity, and Criterion

$$
\text { Condition Accuracy Sensitivity More Attractive Criterion Less Attractive Criterion }
$$

\begin{tabular}{lllll}
\hline Study $2 \mathrm{a}$ & & & & \\
\cline { 1 - 2 } Control $(N=545)$ & $67.5 \%(8.5)$ & $1.01(.54)$ & $-.12(.45)$ & $.01(.46)$ \\
Accountability $(N=554)$ & $67.3 \%(8.9)$ & $1.01(.57)$ & $-.10(.45)$ & $.04(.49)$ \\
Confirmation Bias $(N=548)$ & $68.1 \%(8.5)$ & $1.05(.55)$ & $-.11(.44)$ & $.02(.48)$ \\
Delay $(N=530)$ & $69.4 \%(7.7)$ & $1.13(.52)$ & $-.16(.44)$ & $.05(.44)$ \\
Implementation Intentions $(N=507)$ & $67.6 \%(8.9)$ & $1.01(.57)$ & $-.05(.42)$ & $-.04(.44)$ \\
Objectivity $(N=500)$ & $67.1 \%(8.8)$ & $1.00(.56)$ & $-.04(.44)$ & $.04(.49)$ \\
Reward $(N=538)$ & $67.7 \%(8.3)$ & $1.04(.54)$ & $-.07(.46)$ & $.05(.48)$ \\
Study $2 \mathrm{~b}$ & & & & $-.004(.47)$ \\
\hline Control $(N=343)$ & $66.3 \%(7.7)$ & $.92(.47)$ & $-.16(.40)$ & $.001(.46)$ \\
Accountability $(N=395)$ & $65.9 \%(9.4)$ & $.90(.56)$ & $-.16(.42)$ & $-.003(.45)$ \\
Confirmation Bias $(N=367)$ & $66.5 \%(10.0)$ & $.95(.62)$ & $-.11(.40)$ & $-.02(.40)$ \\
Delay $(N=402)$ & $69.1 \%(7.8)$ & $1.08(.49)$ & $-.21(.38)$ & $-.03(.44)$ \\
Implementation Intentions $(N=338)$ & $66.4 \%(8.5)$ & $.94(.53)$ & $-.10(.46)$ & $.004(.43)$ \\
Objectivity $(N=439)$ & $66.7 \%(8.2)$ & $.96(.51)$ & $-.09(.45)$ & $-.12(.43)$ \\
Reward $(N=375)$ & $66.9 \%(9.3)$ & $.97(.57)$ & \\
\hline
\end{tabular}


Intuitions and Reducing Discrimination

Table 3. Within-Subjects $t$-Tests for Criterion Bias in Each Condition
Condition
Test Statistic
d $\quad 95 \% \mathrm{CI}$

\begin{tabular}{|c|c|c|c|}
\hline Study $2 a$ & & & \\
\hline Control & $t(544)=5.89, p<.001$ & .25 & {$[.17, .34]$} \\
\hline Accountability & $t(553)=6.62, p<.001$ & .28 & {$[.20, .37]$} \\
\hline Confirmation Bias & $t(547)=4.46, p<.001$ & .19 & {$[.11, .27]$} \\
\hline Delay & $t(529)=6.40, p<.001$ & .28 & {$[.19, .36]$} \\
\hline Implementation Intentions & $t(506)=1.00, p=.317$ & .04 & {$[-.04, .13]$} \\
\hline Objectivity & $t(499)=4.20, p<.001$ & .19 & {$[.10, .28]$} \\
\hline Reward & $t(537)=6.66, p<.001$ & .29 & {$[.20, .37]$} \\
\hline Study $2 b$ & & & \\
\hline Control & $t(342)=6.33, p<.001$ & .34 & {$[.23, .45]$} \\
\hline Accountability & $t(394)=6.88, p<.001$ & .35 & {$[.24, .45]$} \\
\hline Confirmation Bias & $t(366)=5.33, p<.001$ & .28 & {$[.17, .38]$} \\
\hline Delay & $t(401)=8.59, p<.001$ & .43 & {$[.33, .53]$} \\
\hline Implementation Intentions & $t(337)=3.32, p=.001$ & .18 & {$[.07, .29]$} \\
\hline Objectivity & $t(348)=4.98, p<.001$ & .27 & {$[.16, .37]$} \\
\hline Reward & $t(375)=5.59, p<.001$ & .29 & {$[.19, .39]$} \\
\hline
\end{tabular}


Intuitions and Reducing Discrimination

Table 4. Independent Samples $t$-Tests for Criterion Bias and Sensitivity Versus Control
Condition
Criterion Bias
Sensitivity

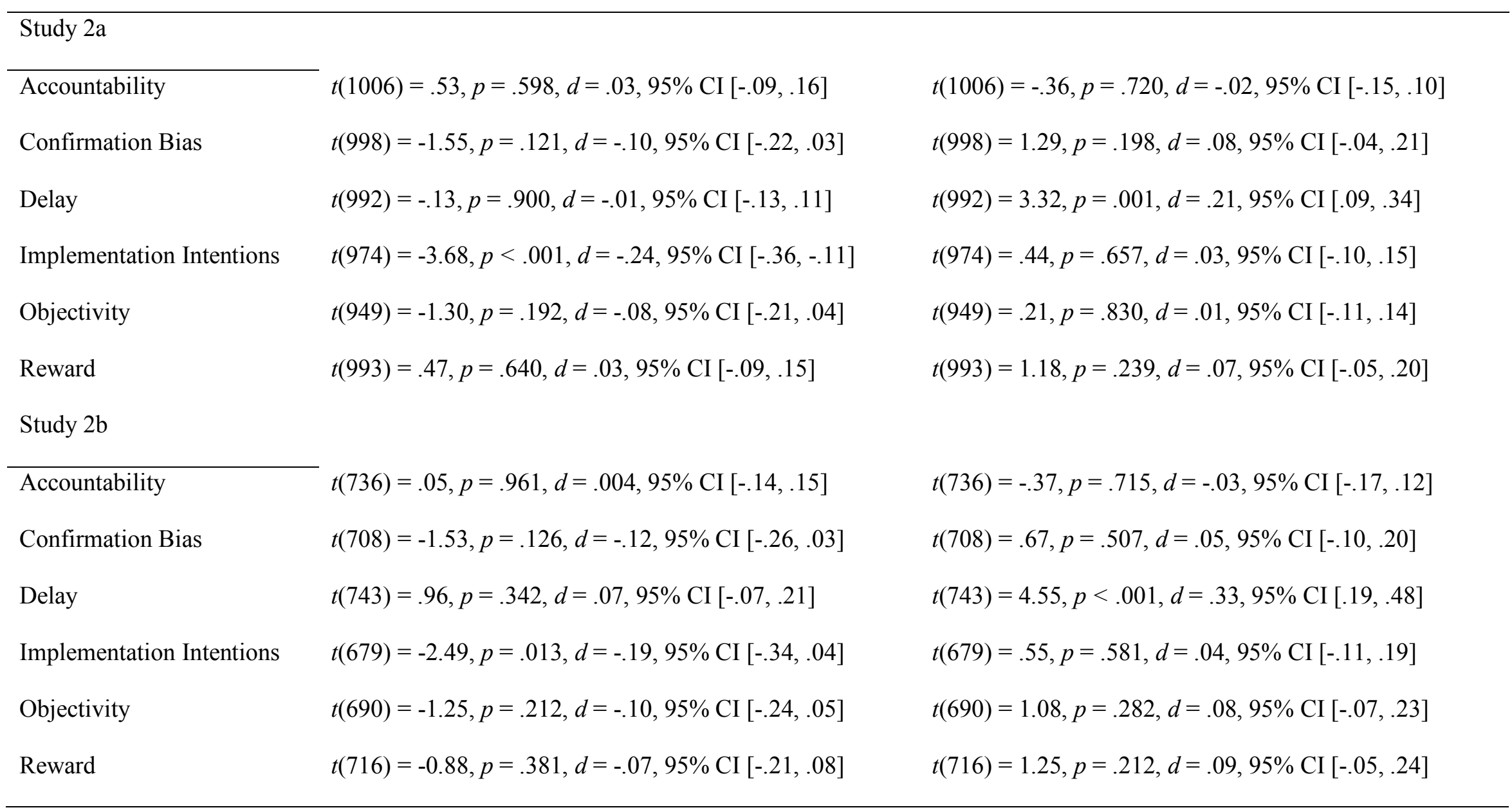

